\title{
Increased Inclusion of Dietary Methionine Sources Improves Pork Quality in Association with the Regulation of Energy and Protein Metabolism and Muscle's Fiber Profile
}

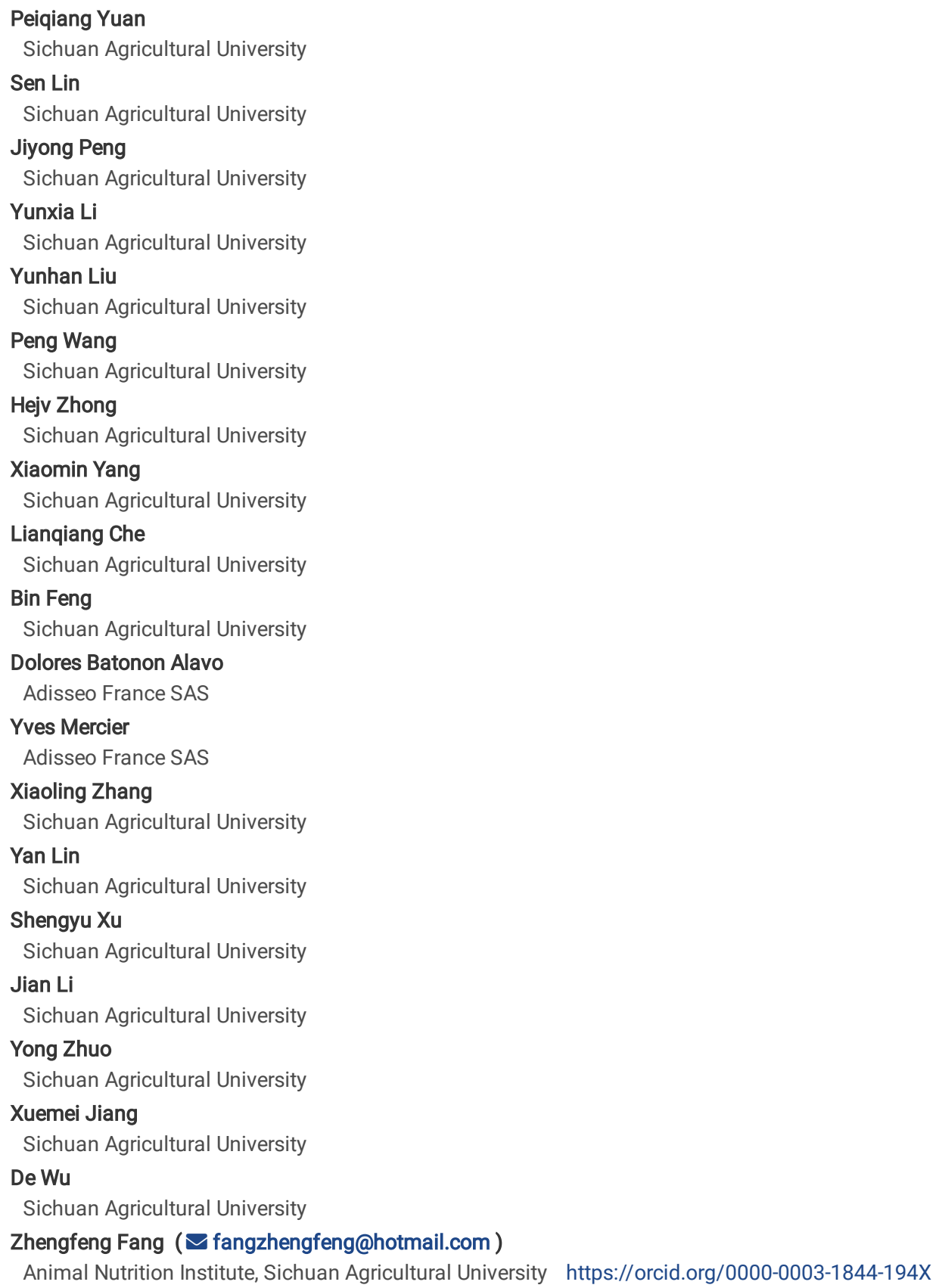




\section{Abstract \\ Background}

Intensive selection for faster growth rate and higher lean percentage led to increase in protein deposition but deterioration in meat quality of pigs, thus there is growing interest in exploring the nutritional strategies to improve meat quality. Methionine has been shown to activate mechanistic target of rapamycin complex 1 protein kinase that plays pivotal roles in the regulation of protein and lipid synthesis. However, few study reports are available regarding the effects of dietary methionine supplementation at levels beyond growth requirements on lipid and protein metabolism and thus on pork quality. The objective of this study was to assess whether pork quality was improved by increasing dietary digestible sulfur amino acids (SAA) levels, with pigs fed the control (100\% SAA), DL-Methionine (125\% SAA)- or OH-Methionine (125\% SAA)-supplemented diets during 11-110 kg period.

\section{Results}

Increasing SAA above requirements did not significantly affect growth performance, whereas improved pork quality as indicated by the decreased drip loss and a tendency towards decrease in shear force of longissimus lumborum muscle. Moreover, fresh muscle from barrows fed OH-Methionine showed a higher lightness value compared with the control and DL-Methionine treatments. The relatively lower shear force might be explained by the decrease in crude protein and increase in glycolytic potential, while the decreased drip loss was associated with down-regulation of genes (like fast glycolytic llx) regulating fiber types. The increased lightness value of fresh muscle from barrows fed OH-Met diets appeared to be associated with the increased lactate level, which can be further explained by the increased plasma short-chain fatty acids concentrations, up-regulated G-protein coupled receptor 43 activation and enhanced glucagon-like peptide 1 secretion.

\section{Conclusion}

Increased SAA consumption appeared to improve pork water-holding capacity and tenderness likely through regulation of energy and protein metabolism and muscle's fiber profile, which provides new insights into the nutritional strategies to improve meat quality.

\section{Introduction}

Intensive selection for greater growth rate and lean percentage led to increase in protein deposition rates but deterioration in meat quality of pigs. Methionine (Met) is considered the second or third limiting amino acids (AA) for the corn-soybean-based swine diets (NRC, 2012) ${ }^{[1]}$. Met is not only a precursor of protein synthesis, but also involved in the methylation cycle, providing essential metabolites or precursors. For example, S-adenosylmethionine (SAM), which acts as mediators or signal molecules, plays important roles in numerous biological processes. A study has shown that the mechanistic target of rapamycin complex 1 (mTORC1) protein kinase can be activated by SAM through SAMTOR sensor ${ }^{[2]}$. The $\mathrm{mTORC}$ plays pivotal roles in the regulation of anabolism including protein and DNA synthesis. Moreover, there is evidence that SAM stimulates the expression of the genes linked to fatty acid metabolism by suppressing Wnt/ $\beta$-catenin and Hedgehog signaling pathways in porcine muscle satellite cells ${ }^{[3]}$. Simultaneously, a previous in vitro study has shown that a severe deficiency in Met and cysteine (Cys) decreases both the proliferation and differentiation, whereas a mild deficiency only alters the differentiation in porcine preadipocytes ${ }^{[4]}$. These results suggested the potential regulation of dietary Met on protein and lipid metabolism. However, few study reports are available regarding the effects of dietary Met supplementation at levels beyond growth requirements on lipid and protein metabolism and thus on the meat quality in growing-finishing pigs.

DL-Met and its hydroxy analogue, DL-2-hydroxy-4 (methylthio) butanoic acid (OH-Met), have long been used as supplemental Met sources in commercial livestock production. Interestingly, our previous studies suggested that $\mathrm{OH}-\mathrm{Met}$ might be considered as more than a Met precursor, given that pigs fed the $\mathrm{OH}$ Met had a higher acetate concentration in plasma ${ }^{[5]}$ than DL-Met-fed pigs. Acetate has been shown to play an important role in insulin signaling and lipid metabolism ${ }^{[6]}$. In the present study, we hypothesized that methionine levels and sources potentially modified meat quality through regulation of protein and lipid metabolism. To test this hypothesis, both genders of pigs (castrated males vs. females) throughout the growing-finishing period were fed diets supplemented with DL-Met or OH-Met to contain digestible sulfur amino acids (SAA) levels above the growth requirement, and biochemical indices for blood and muscle samples and expression of genes in relation with lipid metabolism and muscle's fibers profile for muscle samples were measured.

\section{Material And Methods Study design, diets and animals}

The study was conducted in a randomized complete block design. A total of 144 (half gilts) crossbred (Duroc $\times$ Large White $\times$ Landrace) barrows and gilts $(11.16 \pm 0.23 \mathrm{~kg}, \sim 5$ weeks of age) selected from a single weanling batch were blocked by gender and bodyweight (BW) and allocated to 36 pens ( 4 piglets/pen) located in the same room.

Piglets in each block were randomly fed one of the three experimental diets (Additional file 1-1). The basal diet, fed to the control (CON) group of pigs, was formulated to meet swine nutrition requirements of NRC (2012 ${ }^{[1]}$. The DL-Met and OH-Met diets were formulated to contain $125 \%$ of standard-ileal-digestible (SID) SAA, $25 \%$ of which, namely, the portion of methionine equivalence beyond the CON level, was supplied by DL-Met ( $99 \%)$ and OH-Met ( $88 \%$, Rhodimet ${ }^{8}$ AT88 from Adisseo Life Science, Shanghai, China), respectively. The experimental period consisted of five phases with pigs fed the experimental diets from $11 \mathrm{~kg}$ to $110 \mathrm{~kg}$ of BW. In each rearing phase from 11 to $70 \mathrm{~kg} \mathrm{BW}$, the control diets, with supplemental DL-Met (99\%), were formulated to contain $100 \%$ of 
SID-SAA as recommended by NRC (2012) ${ }^{[1]}$. Each diet was replicated 12 times with 6 pens of gilts and barrows, respectively. All pigs had free access to pelleted feed and water throughout the experimental period.

\section{Analysis of dietary composition}

The dry matter content of the experimental diets was determined by drying at $105^{\circ} \mathrm{C}$ for $4 \mathrm{~h}$. This procedure was repeated again until diets were dried to constant weight. Crude protein, ether extract, ash and crude fiber in diets were analyzed according to procedures described by AOAC (2000) ${ }^{[7]}$. Amino acids in hydrolyzed feed were analyzed by ion-exchange chromatography using an L8900 high-speed AA analyser (Hitachi, Tokyo, Japan). The Cys and Met contents were determined as cysteic acid and Met sulphone, respectively, after performic acid oxidation before hydrolysis. OH-Met in diet was determined by using the HPLC method as previously described ${ }^{[8]}$. The analyzed nutrient levels in experimental diets are shown in Additional file 1-2.

\section{Blood and tissues sampling}

At the end of each phase, one pig per pen was used for blood sample collection. After overnight fasting (12 h), blood samples were collected via jugular venipuncture by using disposable sterilized syringe and then injected into disposable vacuum tube. Samples were centrifuged at $2550 \times \mathrm{g}$ for 15 min at $4{ }^{\circ} \mathrm{C}$, plasma and serum were collected and stored at $-20^{\circ} \mathrm{C}$ until analyzed.

Samples of longissimus lumborum muscle (LM) were taken within 20 min postmortem from the left side carcass at the last rib, cut into small pieces and snap frozen in liquid nitrogen, and then stored at $-80^{\circ} \mathrm{C}$ for analysis of genes expression, glycogen content and glycolytic potential. In addition, the LM and liver samples $(\sim 100 \mathrm{~g})$ were taken and frozen at $-20^{\circ} \mathrm{C}$ for chemical analysis.

\section{Hormone determination}

Plasma insulin growth factor 1 (IGF-1) and glucagon-like peptide 1 (GLP-1) were measured by using the ELISA kits specific for porcine IGF-1 (EK1127, Signalway Antibody) and GLP-1 (Nanjing Jiancheng Biochemical Institute, Nanjing, China), according to the manufacture's protocol. The levels of IGF-1 and GLP-1 were calculated from the standard curve.

\section{Short-chain fatty acids and free amino acids analysis}

Short-chain fatty acids (SCFA) including acetate and propionate in serum were determined by CP-3800 gas chromatography (Varian, Inc., USA) equipped with a micro-injector $(10 \mu \mathrm{L})$, a flame ionization detector and a capillary chromatographic column (CP-FFAP, $25 \mathrm{~m} \times 0.32 \mathrm{~mm} \times 0.3 \mu \mathrm{m}$ ), as we previously described $^{[9]}$.

Free AA in plasma samples were analyzed as described ${ }^{[10]}$. Briefly, $350 \mu$ l of the sample and $700 \mu$ of $10 \%$ (w/v) sulfonyl salicylic acid solution were mixed thoroughly and centrifuged at $12000 \mathrm{~g}$ for $15 \mathrm{~min}$. Then, the supernatant was collected and analyzed for AA by ion-exchange chromatography using an L8800 high-speed AA analyser (Hitachi, Tokyo, Japan).

\section{Measurement of carcass traits and meat quality}

At the end of the experiment, one pig with average BW $(\sim 110 \mathrm{~kg})$ was selected from each pen and slaughtered for measurement of carcass and meat quality. After $24 \mathrm{~h}$ fasting, pigs were anesthetized by electrical stunning and killed by exsanguination. Live weight at slaughter, carcass weight, dressing percentage, average backfat depth, LM area and carcass length were measured immediately postmortem according to the Chinese guidelines on technical regulation for testing of carcass traits in lean-type pig (NY/T 825-2004, 2004) ${ }^{[11]}$. Backfat depth is the average of measurements at three points: the first rib, the last rib, and the last lumbar vertebra. The percentage of lean meat was calculated as previously described ${ }^{[12]}$.

The LM samples were used to measure marbling score, drip loss, pH value, shear force, muscle color and cook loss. The measurements were performed in the following order: 1) 4.0-cm-thick chop used for drip loss measurement between the 3rd and 4th last rib; 2) Assessment of pH value, marbling score and muscle color at the last rib; 3) 6.0-cm-thick chop used for shear force measurement between the first lumbar and the last lumbar.

Initial ( $\mathrm{pH} 45 \mathrm{~min}$ ) and final $\mathrm{pH}(\mathrm{pH} 24 \mathrm{~h}$ ) were measured in triplicate at $45 \mathrm{~min}$ and $24 \mathrm{~h}$ after slaughter, respectively, by using a hand-held pH meter (pH-STAR, SFK-Technology, Denmark). Meat color was measured using a CR-400 Chroma Meter (MinoltaLtd., Milton Keynes, Japan). Meat color values ( $L^{\star}=$ lightness, $a^{*}=$ redness, $b^{*}=$ yellowness) determined at $45 \mathrm{~min}$ and $24 \mathrm{~h}$ postmortem. Marbling score was determined according to National Pork Producers Council guidelines (NPPC, 1999 ${ }^{[13]}$. Drip loss, cooking loss and shear force were determined as previously described ${ }^{[14]}$. Drip loss and cooking loss were expressed as the weight change percentage. Warner-Bratzler shear force of cooked meat was determined using a Texture Analyzer (TA.XT. Plus, Stable Micro Systems, Godalming, UK).

\section{Muscle and liver chemical composition}

To determine the moisture content in LM and liver tissues, samples were freeze-dried for $72 \mathrm{~h}$ and then dried to a constant weight at $105^{\circ} \mathrm{C}$. Crude protein and ether extract were analyzed according to procedures as described AOAC (2000) ${ }^{[7]}$. Intramuscular fat (IMF) in LM was determined as ether extract and calculated by weight loss.

About one gram of frozen LM samples were homogenized for measurement of glycogen content and glycolytic potential. The glycolytic potential was calculated as the following equation: glycolytic potential $=2 \cdot$ (glycogen content + glucose content + glucose-6-phosphate content) + lactate content. Glycogen and lactate content were measured by using commercial kits (Nanjing Jiancheng Biochemical Institute, Nanjing, China). Glucose content in muscle was determined by using enzymatic methods (GAHK-20, Sigma-Aldrich Inc., St. Louis, MO). The glycolytic potential was expressed as micromoles of equivalent lactate per gram of wet tissue. 


\section{Cross sectional area (CSA) determination}

According to the procedures described in detail ${ }^{[14]}$, the LM samples for CSA determination were prepared by classic hematoxylin and eosin (HE) staining, and the CSA of myofiber was measured by using image processing software (Image-Pro Plus 6.0, Silver Spring, MD, USA).

\section{Antioxidant capacity}

The content of total glutathione (T-GSH; number S0053), glutathione disulfide (GSSG, number S0053), glutathione peroxidase (GPX, number S0056) and malondialdehyde (MDA; number S0131) in muscle were analyzed by using commercial kits (Beyotime Biotechnology, Shanghai, China). The concentrations of protein carbonyl (PC; number BC1275) were measured by using commercial kits (Beijing Solarbio Science \& Technology Co., Ltd). Frozen LM samples (

$150 \mathrm{mg}$ ) were quickly removed and homogenized (wt/vol) with ice-cold extracting solution using homogenizer and then centrifuged at $4{ }^{\circ} \mathrm{C}$. The supernatant was then collected and immediately used for further analysis. All operations were carried out according to the instructions provided with the kits.

\section{RNA extraction and real-time PCR}

LM samples were used to determine the expression of genes in association with energy and lipids metabolism, the IGF-1 pathway and the muscle fiber type. These genes include the fatty acid transport protein - 1 (FATP-1), the fatty acid synthase (FAS), the hormone-sensitive lipase (HSL), the AMP-activated protein kinase a2 (AMPKa2), the glucose transporter-4 (GLUT-4), the G protein-coupled receptor (GPR) 43, IGF-1, IGF-1 receptors (IGF-1R) and the Myosin Heavy Chain

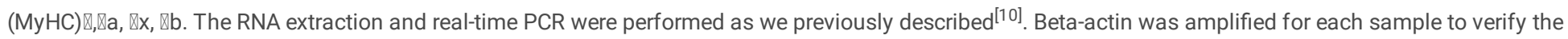
presence of $c D N A$ and as an internal control to calculate the relative level of target gene expression using the $2^{-\triangle \Delta C T}$ method $^{[15]}$. The primer sequences are shown in Additional file 2 .

\section{Statistical analysis}

Data were analyzed by using the mixed model procedure of SAS statistical package (V8.1, SAS Institute Inc., Cary, NC, USA), according to the following equation

$Y_{i j k}=\mu+a_{i}+\beta_{j}+(a \beta)_{i j}+\varepsilon_{i j}$

where $Y$ is the response variable, $\mu$ is the intercept, $a_{i}$ is the effect of treatment $(i=1,2,3), \beta_{j}$ is the effect of gender $(j=1,2),(a \beta)_{i j}$ refers to the interaction between treatment and gender, and $\varepsilon_{\mathrm{ij}}$ represents the residual error. The pen was used as the experimental unit. When an effect was significant $(P<0.05)$, the Tukey test was used to determine specific differences between means.

\section{Results}

\section{Growth Performance}

The BW at the beginning and at the end of each experimental phase was significantly $(P<0.05)$ lower in gilts than in barrows (Table 1$)$. The average daily gain $(A D G)$ and average daily feed intake (ADFI) were also lower $(P<0.05)$ in gilts than in barrows at each of the experimental phases from $25 \mathrm{~kg}$ to $110 \mathrm{~kg}$. The ratio of gain to feed (G:F) was higher $(P<0.05)$ in gilts than in barrows during the $45-70 \mathrm{~kg}, 70-95 \mathrm{~kg}$, and $11-110 \mathrm{~kg}$ periods. The increase of Met above requirements did not significantly $(P>0.05)$ affect the $A D G, A D F I$ and $G$ :F throughout the experimental period. 
Table 1

Effects of dietary treatment and sex on growth performance of growing-finishing pigs

\begin{tabular}{|c|c|c|c|c|c|c|c|c|c|c|c|c|}
\hline \multirow[t]{2}{*}{ Item } & \multicolumn{2}{|l|}{ CON } & \multicolumn{2}{|l|}{ DL-Met } & \multicolumn{2}{|l|}{ OH-Met } & \multicolumn{3}{|l|}{ Diet } & \multicolumn{2}{|l|}{ Sex } & \multirow[t]{2}{*}{$S$} \\
\hline & Barrow & Gilt & Barrow & Gilt & Barrow & Gilt & CON & $\begin{array}{l}\text { DL- } \\
\text { Met }\end{array}$ & $\begin{array}{l}\mathrm{OH}- \\
\text { Met }\end{array}$ & Barrow & Gilt & \\
\hline $\begin{array}{l}\text { Initial } \\
\text { bodyweight } \\
\text { (BW) }\end{array}$ & 11.38 & 10.95 & 11.38 & 10.95 & 11.37 & 10.95 & 11.16 & 11.16 & 11.16 & $11.38^{a}$ & $10.95^{b}$ & 0 \\
\hline \multicolumn{13}{|l|}{$\begin{array}{l}\text { Phase } \\
\text { 1(week1-4) }\end{array}$} \\
\hline $\mathrm{BW}^{1}, \mathrm{~kg}$ & 23.28 & 22.96 & 23.51 & 22.59 & 23.53 & 22.70 & 23.12 & 23.05 & 23.11 & $23.44^{\mathrm{a}}$ & $22.75^{\mathrm{b}}$ & 0 \\
\hline $\mathrm{ADG}^{2}, \mathrm{~g} / \mathrm{d}$ & 425 & 429 & 433 & 416 & 434 & 420 & 427 & 425 & 427 & 431 & 421 & 2 \\
\hline$A D F I^{3}, g / d$ & 679 & 679 & 693 & 677 & 692 & 671 & 679 & 685 & 681 & 688 & 675 & 3 \\
\hline $\mathrm{G}: \mathrm{F}^{4}$ & 0.626 & 0.631 & 0.625 & 0.614 & 0.627 & 0.626 & 0.628 & 0.620 & 0.627 & 0.626 & 0.624 & 0 \\
\hline \multicolumn{13}{|l|}{$\begin{array}{l}\text { Phase } \\
\text { 2(week5-9) }\end{array}$} \\
\hline BW,kg & 45.33 & 42.88 & 43.79 & 42.42 & 45.25 & 42.33 & 44.10 & 43.10 & 43.79 & $44.79^{a}$ & $42.54^{\mathrm{b}}$ & 2 \\
\hline$A D G, g / d$ & 630 & 569 & 579 & 566 & 621 & 561 & 600 & 573 & 591 & $610^{a}$ & $566^{b}$ & 6 \\
\hline$A D F I, g / d$ & 1464 & 1312 & 1350 & 1265 & 1390 & 1268 & 1388 & 1308 & 1329 & $1401^{a}$ & $1282^{b}$ & 1 \\
\hline $\mathrm{G}: \mathrm{F}$ & 0.430 & 0.433 & 0.428 & 0.448 & 0.447 & 0.442 & 0.432 & 0.438 & 0.444 & 0.435 & 0.441 & 0 \\
\hline \multicolumn{13}{|l|}{$\begin{array}{l}\text { Phase } \\
\text { 3(week10- } \\
\text { 13) }\end{array}$} \\
\hline BW,kg & 68.13 & 64.67 & 66.71 & 63.21 & 68.21 & 61.67 & 66.40 & 64.96 & 64.94 & $67.68^{a}$ & $63.18^{b}$ & 4 \\
\hline$A D G, g / d$ & 814 & 778 & 818 & 743 & 820 & 690 & 796 & 781 & 755 & $817^{a}$ & $737^{b}$ & 7 \\
\hline$A D F I, g / d$ & 2304 & 2043 & 2124 & 1918 & 2250 & 1826 & 2173 & 2021 & 2038 & $2226^{a}$ & $1929^{b}$ & 1 \\
\hline $\mathrm{G}: \mathrm{F}$ & 0.353 & 0.381 & 0.385 & 0.387 & 0.365 & 0.379 & 0.368 & 0.386 & 0.372 & $0.368^{b}$ & $0.382^{a}$ & 0 \\
\hline \multicolumn{13}{|l|}{$\begin{array}{l}\text { Phase } \\
\text { 4(week14- } \\
\text { 17) }\end{array}$} \\
\hline BW ,kg & 95.13 & 86.75 & 90.88 & 85.83 & 94.04 & 84.46 & 90.94 & 88.35 & 89.25 & $93.35^{a}$ & $85.68^{b}$ & 6 \\
\hline$A D G, g / d$ & 964 & 789 & 863 & 808 & 923 & 814 & 876 & 836 & 868 & $917^{a}$ & $804^{b}$ & 9 \\
\hline$A D F I, g / d$ & 2888 & 2378 & 2719 & 2362 & 2814 & 2348 & 2633 & 2541 & 2581 & $2807^{a}$ & $2363^{b}$ & 2 \\
\hline $\mathrm{G}: \mathrm{F}$ & 0.334 & 0.331 & 0.317 & 0.341 & 0.328 & 0.346 & 0.333 & 0.329 & 0.337 & $0.326^{\mathrm{b}}$ & $0.339^{a}$ & 0 \\
\hline \multicolumn{13}{|l|}{$\begin{array}{l}\text { Phase } \\
5(\text { week18- } \\
\text { 20) }\end{array}$} \\
\hline BW,kg & 114.33 & 103.54 & 111.25 & 101.79 & 114.46 & 101.54 & 108.94 & 106.52 & 108.00 & $113.35^{a}$ & $102.29^{b}$ & 8 \\
\hline$A D G, g / d$ & 915 & 800 & 970 & 760 & 972 & 813 & 857 & 865 & 893 & $952^{a}$ & $791^{b}$ & 1 \\
\hline$A D F I, g / d$ & 2909 & 2571 & 3054 & 2380 & 3176 & 2550 & 2740 & 2717 & 2863 & $3046^{a}$ & $2501^{b}$ & 3 \\
\hline $\mathrm{G}: \mathrm{F}$ & 0.315 & 0.311 & 0.315 & 0.319 & 0.306 & 0.319 & 0.313 & 0.317 & 0.313 & 0.312 & 0.317 & 0 \\
\hline
\end{tabular}

${ }^{\mathrm{ab}}$ Means within a row with no common superscripts are significantly different $(P<0.05)$.

${ }^{1} \mathrm{BW}$ : Body weight; ${ }^{2} \mathrm{ADG}$ : Average daily gain; ${ }^{3} \mathrm{ADFl}$ : Average daily feed intake; ${ }^{4} \mathrm{G}: \mathrm{F}$ : Gain : feed. 


\begin{tabular}{|c|c|c|c|c|c|c|c|c|c|c|c|c|}
\hline \multirow[t]{2}{*}{ Item } & \multicolumn{2}{|l|}{ CON } & \multicolumn{2}{|l|}{ DL-Met } & \multicolumn{2}{|l|}{ OH-Met } & \multicolumn{3}{|l|}{ Diet } & \multicolumn{2}{|l|}{ Sex } & \multirow[t]{2}{*}{$S$} \\
\hline & Barrow & Gilt & Barrow & Gilt & Barrow & Gilt & CON & $\begin{array}{l}\text { DL- } \\
\text { Met }\end{array}$ & $\begin{array}{l}\mathrm{OH}- \\
\text { Met }\end{array}$ & Barrow & Gilt & \\
\hline \multicolumn{13}{|c|}{$\begin{array}{l}\text { Total } \\
\text { Phase } \\
\text { (week1-20) }\end{array}$} \\
\hline$A D G, g / d$ & 735 & 661 & 713 & 649 & 736 & 643 & 698 & 681 & 690 & $728^{a}$ & $651^{b}$ & 5 \\
\hline $\mathrm{ADFl}, \mathrm{g} / \mathrm{d}$ & 1977 & 1733 & 1903 & 1665 & 1975 & 1668 & 1855 & 1784 & 1822 & $1951^{a}$ & $1689^{b}$ & 1 \\
\hline $\mathrm{G}: \mathrm{F}$ & 0.372 & 0.381 & 0.374 & 0.390 & 0.373 & 0.385 & 0.377 & 0.382 & 0.379 & $0.373^{b}$ & $0.385^{a}$ & 0 \\
\hline \multicolumn{13}{|c|}{ ab Means within a row with no common superscripts are significantly different $(P<0.05)$. } \\
\hline
\end{tabular}

\section{Plasma IGF-1, GLP-1 and short-chain fatty acids concentrations}

Plasma IGF-1 and GLP-1 concentrations both were significantly $(P<0.05$ and $P<0.01$, respectively) affected by dietary treatment. Specifically, plasma IGF-1 concentrations were lower $(P<0.05)$ in the $\mathrm{OH}-\mathrm{Met}$ than in the CON treatment, whereas plasma GLP-1 concentrations were higher $(P<0.05)$ in the OH-Met than in the CON and DL-Met treatments (Table 2). The OH-Met treatment showed higher $(P<0.05)$ serum propionate concentrations and a tendency $(P=0.086)$ towards increase in serum acetate concentrations compared with the CON and DL-Met treatments (Table 2).

Table 2

Effects of dietary treatment and sex on blood indicator at the end of experimental period

\begin{tabular}{|c|c|c|c|c|c|c|c|c|c|c|c|c|}
\hline \multirow[t]{2}{*}{ Item } & \multicolumn{2}{|l|}{ CON } & \multicolumn{2}{|l|}{ DL-Met } & \multicolumn{2}{|l|}{ OH-Met } & \multicolumn{3}{|l|}{ Diet } & \multicolumn{2}{|l|}{ Sex } & \multirow[t]{2}{*}{ SEM } \\
\hline & Barrow & Gilt & Barrow & Gilt & Barrow & Gilt & CON & DL-Met & $\begin{array}{l}\mathrm{OH}- \\
\text { Met }\end{array}$ & Barrow & Gilt & \\
\hline $\begin{array}{l}\text { IGF-1 }{ }^{1} \\
\mathrm{pg} / \mathrm{ml}\end{array}$ & 207.6 & 256.1 & 209 & 213.3 & 167 & 201.8 & $231.9^{a}$ & $211.2^{a b}$ & $184.4^{\mathrm{b}}$ & 194.5 & 223.8 & 45.5 \\
\hline $\begin{array}{l}\text { GLP-12 } \\
\mathrm{pmol} / \mathrm{I}^{2}\end{array}$ & 3 & 2.5 & 3.2 & 4.5 & 6.8 & 6.3 & $2.8^{b}$ & $3.8^{b}$ & $6.5^{\mathrm{a}}$ & 4.3 & 4.4 & 2.3 \\
\hline $\begin{array}{l}\text { Acetate, } \\
\mu \mathrm{mol} / \mathrm{L}\end{array}$ & 137.4 & 136.9 & 139.2 & 148.2 & 187.1 & 197.9 & $137.2^{b}$ & $143.7^{a b^{*}}$ & $192.5^{\mathrm{a}}$ & 154.6 & 161 & 64.2 \\
\hline $\begin{array}{l}\text { Propionate, } \\
\mu \mathrm{mol} / \mathrm{L}\end{array}$ & 29.4 & 26.1 & 26.7 & 33.3 & 39.5 & 53 & $27.7^{b}$ & $30.0^{\mathrm{b}}$ & $46.3^{a}$ & 31.9 & 37.5 & 18.4 \\
\hline
\end{tabular}

\section{Plasma free amino acids concentrations}

Increasing dietary SAA contributed to variance of plasma amino acids profile in different experimental periods (Table 3, Additional file 3). Notably, plasma taurine concentration showed an increase $(P<0.05$ or $P<0.10)$ following increased SAA consumption as compared with the CON at $11-70 \mathrm{~kg}$ period, whereas showed no difference $(P>0.10)$ among treatments at $70-110 \mathrm{~kg}$ period (Table 3$)$. In contrast, plasma phenylalanine concentration was higher $(P<0.05)$ in CON than in increased SAA treatment at 70-95 kg period, whereas was lower $(P<0.05)$ in CON than in increased SAA treatment at $11-70 \mathrm{~kg}$ period $($ Table 3$)$. 
Table 3

Effects of dietary methionine levels and source on plasma free amino acids concentrations $(\mathrm{ng} / \mu \mathrm{L})$

\begin{tabular}{|c|c|c|c|c|c|c|c|c|c|c|c|c|}
\hline \multirow[t]{2}{*}{ Item } & \multicolumn{2}{|l|}{ CON } & \multicolumn{2}{|l|}{ DL-Met } & \multicolumn{2}{|l|}{ OH-Met } & \multicolumn{3}{|l|}{ Diet } & \multicolumn{2}{|l|}{ Sex } & \multirow[t]{2}{*}{ SEM } \\
\hline & Barrow & Gilt & Barrow & Gilt & Barrow & Gilt & CON & $\begin{array}{l}\text { DL- } \\
\text { Met }\end{array}$ & $\begin{array}{l}\mathrm{OH}- \\
\text { Met }\end{array}$ & Barrow & Gilt & \\
\hline \multicolumn{13}{|c|}{ Phase 1 (11-25 kg) } \\
\hline Methionine & $3.97^{b}$ & $5.00^{\mathrm{a}}$ & $4.48^{\mathrm{ab}}$ & $3.90^{\mathrm{b}}$ & $4.72^{\mathrm{ab}}$ & $4.03^{\mathrm{ab}}$ & 4.49 & 4.19 & 4.38 & 4.39 & 4.31 & 0.87 \\
\hline Phenylalanine & 7.97 & 9.2 & 12.07 & 12.18 & 10.92 & 11.43 & $8.59^{b}$ & $12.12^{\mathrm{a}}$ & $11.17^{a}$ & 10.32 & 10.9 & 2.96 \\
\hline Cysteine & 1.97 & 2.34 & 3.02 & 2.04 & 1.94 & 1.59 & 2.14 & 2.53 & 1.77 & 2.31 & 1.99 & 0.83 \\
\hline Taurine & 8.64 & 10.36 & 15.58 & 16.16 & 16.57 & 15.6 & $9.50^{\mathrm{b}}$ & $15.87^{a}$ & $16.09^{a}$ & 13.6 & 14 & 3.87 \\
\hline \multicolumn{13}{|c|}{ Phase 2 (25-45 kg) } \\
\hline Methionine & 3.81 & 3.75 & 5.64 & 4.1 & 4.8 & 4.81 & $3.78^{b}$ & $4.87^{a}$ & $4.81^{\mathrm{a}}$ & 4.75 & 4.22 & 0.77 \\
\hline Phenylalanine & $7.61^{\mathrm{c}}$ & $7.90^{c}$ & $10.82^{a}$ & $8.45^{\mathrm{bc}}$ & $9.76^{a b}$ & $9.56^{\mathrm{ab}}$ & $7.76^{\mathrm{b}}$ & $9.63^{a}$ & $9.66^{\mathrm{a}}$ & 9.4 & 8.64 & 1.22 \\
\hline Cysteine & 2.13 & 2.16 & 1.39 & 1.16 & 1.17 & 1.81 & $2.15^{\mathrm{a}}$ & $1.29^{b}$ & $1.35^{\mathrm{b}}$ & 1.56 & 1.71 & 0.85 \\
\hline Taurine & 7.88 & 7.34 & 9.96 & 9.11 & 7.44 & 10.02 & 7.61 & 9.54 & 8.73 & 8.43 & 8.82 & 2.03 \\
\hline \multicolumn{13}{|c|}{ Phase 3 (45-70 kg) } \\
\hline Methionine & $3.45^{\mathrm{c}}$ & $4.40^{\mathrm{ab}}$ & $3.72^{b c}$ & $3.96^{\mathrm{bc}}$ & $4.68^{\mathrm{a}}$ & $3.71^{\mathrm{bc}}$ & 3.93 & 3.84 & 4.19 & 3.95 & 4.02 & 0.61 \\
\hline Phenylalanine & 8.01 & 8.01 & 10.08 & 9.72 & 11.91 & 9.73 & $8.01^{b}$ & $9.90^{\mathrm{a}}$ & $10.82^{\mathrm{a}}$ & 10 & 9.15 & 1.55 \\
\hline Cysteine & 1.04 & 1.8 & 0.83 & 0.92 & 1.03 & 1.58 & 1.42 & 0.86 & 1.25 & 0.97 & 1.43 & 0.38 \\
\hline Taurine & 6.62 & 6.34 & 9.9 & 10.04 & 10.27 & 10 & $6.48^{b}$ & $9.97^{a}$ & $10.14^{\mathrm{a}}$ & 8.93 & 8.79 & 2.01 \\
\hline \multicolumn{13}{|c|}{ Phase 4 (70-95 kg) } \\
\hline Methionine & 4.16 & 3.74 & 4.08 & 4.23 & 3.94 & 4.22 & 3.95 & 4.16 & 4.08 & 4.06 & 4.06 & 0.64 \\
\hline Phenylalanine & 9.92 & 9.86 & 8.42 & 8.25 & 8.62 & 8.46 & $9.89^{\mathrm{a}}$ & $8.34^{\mathrm{b}}$ & $8.54^{\mathrm{b}}$ & 8.99 & 8.86 & 1.58 \\
\hline Cysteine & 1.38 & 0.95 & 1.16 & 1.4 & 1.64 & 1.78 & $1.16^{b}$ & $1.28^{\mathrm{ab}}$ & $1.71^{\mathrm{a}}$ & 1.39 & 1.38 & 0.46 \\
\hline Taurine & 9.4 & 8.89 & 8.57 & 8.13 & 9.05 & 8.97 & 9.15 & 8.35 & 9.01 & 9.01 & 8.66 & 1.64 \\
\hline \multicolumn{13}{|c|}{ Phase 5 (95-110 kg) } \\
\hline Methionine & 3.28 & 3.24 & 2.81 & 2.93 & 3.1 & 3.14 & $3.26^{\mathrm{a}}$ & $2.87^{b}$ & $3.12^{\mathrm{ab}}$ & 3.06 & 3.1 & 0.89 \\
\hline Phenylalanine & 6.3 & 6.42 & 5.39 & 5.61 & 5.81 & 5.88 & 6.36 & 5.5 & 5.85 & 5.83 & 5.97 & 1.34 \\
\hline Cysteine & 1.91 & 1.79 & 1.03 & 0.78 & 1.67 & 1.75 & $1.86^{\mathrm{a}}$ & $0.90^{\mathrm{b}}$ & $1.71^{\mathrm{a}}$ & 1.54 & 1.44 & 0.89 \\
\hline Taurine & 7.18 & 6.58 & 7.55 & 7.79 & 8.98 & 7.38 & 6.88 & 7.67 & 8.18 & 7.9 & 7.25 & 1.58 \\
\hline
\end{tabular}

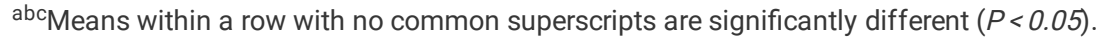

\section{Carcass characteristics and meat quality measured at slaughter}

Dietary treatment showed no effect on $\mathrm{pH}$ values, color parameters or marbling score, whereas significantly $(P<0.05)$ affected drip loss and tended $(P<0.10)$ to affect shear force (Table 4). Compared with the CON treatment, drip loss was decreased $(P<0.05)$ while shear force tended $(P<0.10)$ to decrease in both the DL-Met and OH-Met treatments. The $\mathrm{pH}$ value at $45 \mathrm{~min}$, the color parameter $\mathrm{L}_{45 \mathrm{~min}}$ and drip loss were significantly $(P<0.05)$ affected by the interaction of sex and diet. Interestingly, $\mathrm{pH}_{45 \text { min }}$ value was higher in the OH-Met-fed gilts than in the DL-Met- and CON-fed gilts, while $\mathrm{L}_{45 \mathrm{~min}}$ value was higher in the OH-Metfed barrows than in the DL-Met- and CON-fed barrows. Drip loss was higher $(P<0.05)$ in gilts than in barrows in the CON treatment, whereas it showed no difference $(P>0.10)$ between sex in the DL-Met and OH-Met treatments. Overall, the DL-Met- and OH-Met-fed gilts had lower $(P<0.05)$ drip loss than the CONfed gilts. In addition, compared with gilts, barrows had higher $(P<0.05)$ backfat depth and marbling score values, whereas had lower $(P<0.05)$ lean meat percentage, LM area and shear force. 
Table 4

Effects of dietary treatment and sex on carcass characteristics and meat quality

\begin{tabular}{|c|c|c|c|c|c|c|c|c|c|c|c|c|}
\hline \multirow[t]{2}{*}{ Item } & \multicolumn{2}{|l|}{ CON } & \multicolumn{2}{|l|}{ DL-Met } & \multicolumn{2}{|l|}{ OH-Met } & \multicolumn{3}{|l|}{ Diet } & \multicolumn{2}{|l|}{ Sex } & \multirow[t]{2}{*}{ SEN } \\
\hline & Barrow & Gilt & Barrow & Gilt & Barrow & Gilt & CON & $\begin{array}{l}\text { DL- } \\
\text { Met }\end{array}$ & $\begin{array}{l}\text { OH- } \\
\text { Met }\end{array}$ & Barrow & Gilt & \\
\hline $\begin{array}{l}\text { Final body } \\
\text { weight, kg }\end{array}$ & 108.83 & 105.68 & 109.07 & 104.4 & 111.15 & 109.22 & 107.26 & 106.73 & 110.18 & $109.68^{a}$ & $106.43^{b}$ & 3.89 \\
\hline $\begin{array}{l}\text { Dressing } \\
\text { percentage, } \\
\%\end{array}$ & 78.41 & 77.51 & 77.62 & 78.29 & 78.45 & 77.58 & 77.96 & 77.95 & 78.01 & 78.16 & 77.79 & 1.08 \\
\hline $\begin{array}{l}\text { Lean meat, } \\
\%\end{array}$ & 59.98 & 63.04 & 59.74 & 64.46 & 60.17 & 63.33 & 61.51 & 62.1 & 61.75 & $59.96^{b}$ & $63.61^{a}$ & 2.23 \\
\hline $\begin{array}{l}\text { Yield of cold } \\
\text { carcass, } \%\end{array}$ & 98.39 & 98.37 & 98.51 & 98.46 & 98.47 & 98.51 & 98.38 & 98.49 & 98.49 & 98.46 & 98.45 & 0.16 \\
\hline $\begin{array}{l}\text { Carcass } \\
\text { length, cm }\end{array}$ & 92.5 & 94 & 93.33 & 93.83 & 94.67 & 94.5 & 93.25 & 93.58 & 94.58 & 93.5 & 94.11 & 3.04 \\
\hline $\begin{array}{l}\text { Longissimus } \\
\text { area, } \mathrm{cm}^{2}\end{array}$ & 79.79 & 87.89 & 79.56 & 82.28 & 74.42 & 85.15 & 83.84 & 80.92 & 79.79 & $77.92^{b}$ & $85.11^{a}$ & 7.33 \\
\hline \multicolumn{13}{|l|}{$\begin{array}{l}\text { Backfat } \\
\text { depth, mm }\end{array}$} \\
\hline First rib & 34.9 & 28.27 & 35.01 & 31.2 & 35.59 & 31.19 & 31.58 & 33.1 & 33.39 & $35.17^{a}$ & $30.22^{b}$ & 4.49 \\
\hline Last rib & 25.3 & 18.78 & 23.33 & 19.42 & 22.85 & 18.9 & 22.04 & 21.37 & 20.88 & $23.83^{a}$ & $19.03^{b}$ & 3.56 \\
\hline $\begin{array}{l}\text { Last lumbar } \\
\text { vertebra }\end{array}$ & 18.52 & 14.31 & 20.58 & 14.26 & 18.6 & 15.04 & 16.42 & 17.42 & 16.82 & $19.23^{\mathrm{a}}$ & $14.54^{b}$ & 3.33 \\
\hline $\begin{array}{l}\text { Average } \\
\text { backfat, } \mathrm{mm}\end{array}$ & 26.24 & 20.45 & 26.3 & 21.63 & 25.68 & 21.71 & 23.35 & 23.97 & 23.7 & $26.07^{a}$ & $21.26^{\mathrm{b}}$ & 3.2 \\
\hline $\mathrm{pH}_{45 \text { min }}$ & $6.6^{\mathrm{ab}}$ & $6.4^{\mathrm{b}}$ & $6.6^{a b}$ & $6.4^{\mathrm{b}}$ & $6.5^{\mathrm{b}}$ & $6.9^{a}$ & 6.5 & 6.5 & 6.7 & 6.5 & 6.6 & 0.3 \\
\hline $\mathrm{pH}_{24 \mathrm{~h}}$ & 5.6 & 5.5 & 5.6 & 5.8 & 5.7 & 5.6 & 5.6 & 5.7 & 5.6 & 5.6 & 5.6 & 0.2 \\
\hline \multicolumn{13}{|l|}{$\begin{array}{l}\text { Color } \\
\text { parameters }\end{array}$} \\
\hline $\mathrm{L}_{45 \min ^{1}}{ }^{1}$ & $37.3^{b}$ & $38.1^{\mathrm{ab}}$ & $37.8^{b}$ & $38.1^{\mathrm{ab}}$ & $39.7^{a}$ & $36.9^{b}$ & 37.7 & 38 & 38.3 & 38.3 & 37.7 & 3.5 \\
\hline$a_{45 \min }{ }^{2}$ & 3.3 & 3.6 & 3.2 & 3.1 & 3.1 & 3.5 & 3.5 & 3.1 & 3.3 & 3.2 & 3.4 & 1.5 \\
\hline$b_{45 \min ^{3}}$ & 2.8 & 2.8 & 3 & 3 & 3.1 & 2.9 & 2.8 & 3 & 3 & 3 & 2.9 & 1.2 \\
\hline $\mathrm{L}_{24 \mathrm{~h}}$ & 47.6 & 47.7 & 48.2 & 47.2 & 47.7 & 47.1 & 47.6 & 47.7 & 47.4 & 47.8 & 47.3 & 2.7 \\
\hline$a_{24 h}$ & 4.6 & 5.2 & 4.6 & 4 & 4 & 4.9 & 4.9 & 4.3 & 4.4 & 4.4 & 4.7 & 1 \\
\hline $\mathrm{b}_{24 \mathrm{~h}}$ & 3.5 & 3.8 & 4 & 3.3 & 3.5 & 3.5 & 3.7 & 3.6 & 3.5 & 3.7 & 3.5 & 0.7 \\
\hline $\begin{array}{l}\text { Shear force, } \\
N\end{array}$ & 29.1 & 35.7 & 23.3 & 29.6 & 26.1 & 28.5 & 32.4 & 26.4 & 27.3 & 26.2 & 31.2 & 6.4 \\
\hline $\begin{array}{l}\text { Marbling } \\
\text { score }\end{array}$ & 2.9 & 2.7 & 3 & 2.6 & 3.1 & 2.7 & 2.8 & 2.8 & 2.9 & 3 & 2.6 & 0.4 \\
\hline Drip loss, \% & $1.7^{\mathrm{b}}$ & $2.1^{\mathrm{a}}$ & $1.8^{\mathrm{ab}}$ & $1.6^{\mathrm{b}}$ & $1.6^{\mathrm{b}}$ & $1.6^{\mathrm{b}}$ & $1.9^{\mathrm{a}}$ & $1.7^{\mathrm{b}}$ & $1.6^{\mathrm{b}}$ & 1.7 & 1.8 & 0.2 \\
\hline Cook loss, \% & 31.2 & 29.5 & 28.9 & 28.4 & 31.1 & 28.7 & 30.3 & 28.6 & 29.9 & 30.4 & 28.9 & 5.13 \\
\hline
\end{tabular}

${ }^{\mathrm{ab}}$ Means within a row with no common superscripts are significantly different $(P<0.05)$.

${ }^{1} \mathrm{~L}^{*}=$ lightness; ${ }^{2} \mathrm{a}^{*}=$ redness; ${ }^{3} \mathrm{~b}^{*}=$ yellowness

\section{Protein and fat content in muscle and liver}

The sex of pigs showed a significant $(P<0.05)$ effect on crude protein and IMF content in LM muscles. Overall, the gilts had a higher $(P<0.05)$ crude protein and a lower $(P<0.05)$ IMF content than barrows. In addition, crude protein content in fresh liver tended $(P<0.10)$ to be lower in the DL-Met and OH-Met treatments than in the CON treatment (Table 5). 
Table 5

Muscle and liver composition following consumption of diets adequate or above sulfur amino acids requirements

\begin{tabular}{|c|c|c|c|c|c|c|c|c|c|c|c|c|}
\hline \multirow[t]{2}{*}{ Item } & \multicolumn{2}{|l|}{ CON } & \multicolumn{2}{|l|}{ DL-Met } & \multicolumn{2}{|l|}{ OH-Met } & \multicolumn{3}{|l|}{ Diet } & \multicolumn{2}{|l|}{ Sex } & EM \\
\hline & Barrow & Gilt & Barrow & Gilt & Barrow & Gilt & CON & $\begin{array}{l}\text { DL- } \\
\text { Met }\end{array}$ & $\begin{array}{l}\mathrm{OH}- \\
\text { Met }\end{array}$ & Barrow & Gilt & \\
\hline \multicolumn{13}{|c|}{ Longissimus muscle } \\
\hline Moisture, \% & 73.7 & 73.5 & 73.8 & 73.4 & 74.1 & 73.5 & 73.6 & 73.6 & 73.8 & 73.9 & 73.5 & 0.9 \\
\hline $\begin{array}{l}\text { Crude Protein } \\
\text { in fresh } \\
\text { tissue, } \mathrm{g} / \mathrm{kg}\end{array}$ & 223.7 & 230.2 & 213.8 & 224 & 213.6 & 224.4 & 226.9 & 218.9 & 219.0 & 217 & 226.2 & 10.3 \\
\hline $\begin{array}{l}\text { Intramuscular } \\
\text { fat,\% }\end{array}$ & 3.0 & 2.3 & 2.7 & 2.6 & 3.2 & 2.4 & 2.7 & 2.6 & 2.8 & 3.0 & 2.4 & 0.8 \\
\hline $\begin{array}{l}\text { Lactate, } \\
\mu \mathrm{mol} / \mathrm{g}\end{array}$ & 69.9 & 74 & 76.9 & 75.6 & 82 & 78.5 & $72.0^{\mathrm{b}}$ & $76.2^{\mathrm{ab}}$ & $80.3^{a}$ & 76.2 & 76.1 & 6.3 \\
\hline $\begin{array}{l}\text { Free glucose } \\
\text { and glucose- } \\
6-P, \mu \mathrm{mol} / \mathrm{g}\end{array}$ & 9.9 & 9.8 & 13.4 & 14.5 & 12.6 & 10.6 & $9.8^{b}$ & $13.9^{a}$ & $11.6^{\mathrm{ab}}$ & 11.9 & 11.6 & 3.7 \\
\hline $\begin{array}{l}\text { Glycogen, } \\
\mu \mathrm{mol} / \mathrm{g}\end{array}$ & 5.3 & 5 & 5.6 & 4.8 & 4.7 & 6.6 & 5.1 & 5.2 & 5.7 & 5.2 & 5.4 & 2.3 \\
\hline $\begin{array}{l}\text { Glycolytic } \\
\text { potential, } \\
\mu \mathrm{mol} / \mathrm{g}\end{array}$ & 100.2 & 103.5 & 114.9 & 114.1 & 116.6 & 113 & $101.9^{b}$ & $114.5^{\mathrm{a}}$ & $114.8^{\mathrm{a}}$ & 110.6 & 110.2 & 10.8 \\
\hline $\begin{array}{l}\text { T-GSH }{ }^{1} \text {, } \\
\text { nmol/g } \\
\text { Muscle }\end{array}$ & 298.8 & 301.7 & 260.9 & 282 & 276.5 & 269.6 & 300.2 & 271.5 & 273.0 & 278.7 & 284.4 & 33.1 \\
\hline $\begin{array}{l}\mathrm{GSSG}^{2} \\
\mathrm{nmol} / \mathrm{g} \\
\text { Muscle }\end{array}$ & 52.7 & 52.2 & 51.3 & 49.6 & 51.2 & 45.4 & 52.4 & 50.4 & 48.3 & 51.7 & 49.0 & 21.0 \\
\hline $\begin{array}{l}\mathrm{GSH}^{3}, \mathrm{nmol} / \mathrm{g} \\
\text { Muscle }\end{array}$ & 193.4 & 197.4 & 158.4 & 182.9 & 165.7 & 178.9 & 195.4 & 170.7 & 172.3 & 172.5 & 186.4 & 49.2 \\
\hline GSH/GSSG & 3.5 & 2.8 & 3.4 & 3.2 & 3.2 & 3.5 & 3.2 & 3.3 & 3.4 & 3.4 & 3.2 & 1.6 \\
\hline $\begin{array}{l}\text { GPX }{ }^{4} \\
\text { Units/mg } \\
\text { protein }\end{array}$ & 11.1 & 10.9 & 7.4 & 10.6 & 11 & 10.6 & 11 & 9 & 10.8 & 9.8 & 10.7 & 3.8 \\
\hline $\begin{array}{l}\text { MDA }^{5} \\
\text { nmol/g } \\
\text { Muscle }\end{array}$ & 42.2 & 48.2 & 40.3 & 44.2 & 42 & 43.2 & 46.4 & 42.3 & 42.6 & 41.5 & 46 & 6.1 \\
\hline $\begin{array}{l}\text { Protein } \\
\text { carbonyl, } \\
\text { «mol/mg } \\
\text { protein }\end{array}$ & 0.041 & 0.037 & 0.044 & 0.044 & 0.043 & 0.032 & 0.039 & 0.044 & 0.038 & 0.043 & 0.038 & 0.019 \\
\hline \multicolumn{13}{|l|}{ Liver } \\
\hline Moisture,\% & 71.7 & 71.7 & 72 & 71.4 & 72 & 71.5 & 71.7 & 71.7 & 71.8 & 71.9 & 71.6 & 0.9 \\
\hline $\begin{array}{l}\text { Crude Protein } \\
\text { in fresh } \\
\text { tissue, } \mathrm{g} / \mathrm{kg}\end{array}$ & 211.2 & 207.7 & 203.6 & 202.9 & 199.1 & 206.6 & $209.5^{a}$ & $203.2^{\mathrm{b}}$ & $202.9^{b}$ & 204.7 & 205.7 & 7.2 \\
\hline
\end{tabular}

${ }^{\mathrm{ab}}$ Means within a row with no common superscripts are significantly different $(P<0.05)$.

${ }^{1} \mathrm{~T}-\mathrm{GSH}=$ total glutathione; ${ }^{2} \mathrm{GSH}=$ reduced glutathione; ${ }^{3} \mathrm{GSSG}=$ oxidized glutathione; ${ }^{4} \mathrm{GPX}=$ glutathione peroxidase; ${ }^{5} \mathrm{MDA}=\mathrm{malondialdehyd}$

\section{Glycolytic potential in muscle}

Dietary treatment showed a significant $(P<0.05)$ effect on lactate, free glucose and glucose-6-P, and glycolytic potential of LM muscle. Specifically, compared with the CON treatment, the $\mathrm{OH}-$ Met treatment had higher $(P<0.05)$ lactate content, whereas the DL-Met treatment had increased $(P<0.05)$ free glucose and glucose-6-P content (Table 5). As a result, both OH-Met and DL-Met treatments showed higher $(P<0.05)$ glycolytic potential than the CON treatment.

Oxidation indices in muscle

The oxidation indices, including T-GSH, GSSG, GSH, GSSH/GSSG, GPX, MDA and protein carbonyl in muscle, were not affected $(P>0.10)$ by diet, sex or diet $\times$ sex interaction (Table 5). 
The CSA was decreased $(P<0.05)$ in DL-Met and OH-Met treatments compared with the CON group (Table 6). The sex of pigs showed a significant $(P<0.05)$ effect on AMPKa2 expression and tended $(P<0.10)$ to affect FAS expression in LM muscles. Compared with barrows, gilts had significantly $(P<0.05)$ lower AMPKa2 mRNA abundance while tended $(P<0.10)$ to have lower FAS mRNA abundance. Dietary treatment had a significant $(P<0.05)$ effect on MyHC $\llbracket \mathrm{x}$, GPR43, IGF-1, AMPKa2, GLUT-4 and FATP-1 expression, while tended to affect $(P<0.10)$ HSL expression in LM muscles. Compared with the CON treatment, the DL-Met and OH-Met treatments had decreased $(P<0.05)$ mRNA abundance of MyHC $\forall x$ and IGF-1 while increased $(P<0.05)$ mRNA abundance of FATP-1, GLUT-4 and AMPKa2. The OH-Met and DL-Met treatments tended $(P<0.10)$ to decrease HSL mRNA abundance compared with the CON treatment. The GPR43 mRNA abundance was higher $(P<0.05)$ in the OH-Met than in the CON treatment. In contrast, MyHC凶, MyHC『a, MyHC『b and IGF-1R expression were not affected $(P>0.10)$ by sex, diet or their interactions.

Table 6

Effects of dietary treatment and sex on myofiber cross sectional area and gene expression in muscle

\begin{tabular}{|c|c|c|c|c|c|c|c|c|c|c|c|}
\hline \multirow[t]{2}{*}{ Item } & \multicolumn{2}{|l|}{ CON } & \multicolumn{2}{|l|}{ DL-Met } & \multicolumn{2}{|l|}{ OH-Met } & \multicolumn{3}{|l|}{ Diet } & \multicolumn{2}{|l|}{ Sex } \\
\hline & Barrow & Gilt & Barrow & Gilt & Barrow & Gilt & CON & DL-Met & OH-Met & Barrow & Gilt \\
\hline \multicolumn{12}{|c|}{ Myofiber cross sectional area, $\mu \mathrm{m}^{2}$} \\
\hline & $2266.1^{\mathrm{a}}$ & $2275.2^{\mathrm{a}}$ & $1764.6^{b}$ & $1700.4^{b}$ & $1821.0^{\mathrm{ab}}$ & $2039.5^{\mathrm{ab}}$ & $2270.6^{a}$ & $1732.5^{\mathrm{b}}$ & $1930.2^{b}$ & 1950.6 & 2005.0 \\
\hline \multicolumn{12}{|c|}{ Muscle fiber types ${ }^{1}$} \\
\hline MyHCD & 1.1 & 1.1 & 0.8 & 1.1 & 0.9 & 1.2 & 1.1 & 0.9 & 1.1 & 0.9 & 1.1 \\
\hline MyHC囚a & 5.1 & 3.3 & 4.6 & 3.9 & 4.3 & 4.3 & 4.2 & 4.2 & 4.3 & 4.6 & 3.8 \\
\hline $\mathrm{MyHC} \rrbracket x$ & 1.7 & 1.3 & 1.0 & 1.1 & 1.1 & 1.1 & $1.5^{\mathrm{a}}$ & $1.1^{b}$ & $1.1^{\mathrm{b}}$ & 1.3 & 1.2 \\
\hline $\mathrm{MyHC} \bowtie b$ & 2.3 & 2.2 & 2.0 & 2.1 & 1.8 & 1.8 & 2.3 & 2.0 & 1.8 & 2.0 & 2.0 \\
\hline \multicolumn{12}{|c|}{ IGF-1 system } \\
\hline IGF-12 ${ }^{2}$ & 3.3 & 3.4 & 2.4 & 2.5 & 2.2 & 1.9 & $3.4^{\mathrm{a}}$ & $2.4^{b}$ & $2.0^{\mathrm{b}}$ & 2.6 & 2.6 \\
\hline IGF-1R ${ }^{3}$ & 2.6 & 3.0 & 2.4 & 2.4 & 2.4 & 2.3 & 2.8 & 2.4 & 2.4 & 2.4 & 2.6 \\
\hline \multicolumn{12}{|c|}{ Fat acids metabolism } \\
\hline $\mathrm{HSL}^{4}$ & 5.3 & 5.9 & 4.2 & 4.4 & 4.1 & 4.2 & $5.6^{\mathrm{a}}$ & $4.3^{a b^{*}}$ & $4.2^{b}$ & 4.5 & 4.9 \\
\hline FATP- ${ }^{5}$ & 3.3 & 3.3 & 4.7 & 4.2 & 4.9 & 5.1 & $3.3^{b}$ & $4.4^{\mathrm{a}}$ & $5.0^{\mathrm{a}}$ & 4.3 & 4.2 \\
\hline FAS $^{6}$ & 1.6 & 1.3 & 1.6 & 0.9 & 1.4 & 0.8 & 1.5 & 1.3 & 1.1 & 1.5 & 1.0 \\
\hline GPR43 ${ }^{7}$ & 4.7 & 4.9 & 6.5 & 5.9 & 8.1 & 7.1 & $4.83^{b}$ & $6.18^{\mathrm{ab}}$ & $7.59^{a}$ & 6.4 & 6.0 \\
\hline \multicolumn{12}{|c|}{ Energy metabolism } \\
\hline $\mathrm{AMPKa}^{8}$ & 4.3 & 3.7 & 5.1 & 4.8 & 5.8 & 4.6 & $4.0^{\mathrm{b}}$ & $4.92^{\mathrm{a}}$ & $5.20^{\mathrm{a}}$ & 5.1 & 4.3 \\
\hline GLUT- $4^{9}$ & 1.5 & 1.5 & 2.4 & 1.8 & 1.9 & 1.9 & $1.50^{\mathrm{b}}$ & $2.10^{\mathrm{a}}$ & $1.89^{a}$ & 1.9 & 1.7 \\
\hline
\end{tabular}

${ }^{\text {ab }}$ Means within a row with no common superscripts are significantly different $(P<0.05)$.

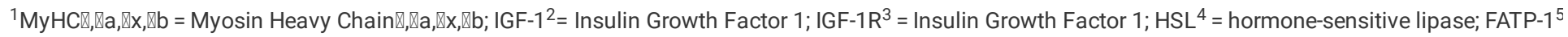

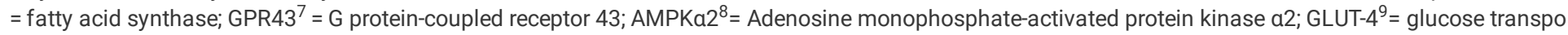

\section{Discussion}

Effects of sex and increased dietary SAA on growth performance and carcass traits

In the present study, barrows showed higher body weight from the beginning until the end of the experiment, which might in part explain the lower growth rate and feed intake in gilts than in barrows during the growing-finishing period. Meanwhile, feed efficiency as indicated by G:F was higher in gilts than in barrows during the $45-70 \mathrm{~kg}, 70-95 \mathrm{~kg}$, and $11-110 \mathrm{~kg}$ periods. These results were in agreement with the previous reports that barrows had higher ADFI and ADG, but lower feed efficiency than gilts ${ }^{[16]}$. The higher feed effeciency of gilts could be explained by the greater protein deposition. In support of this, carcass analysis indicated that barrows had lower lean meat percentage and produced fatter carcass compared with gilts. Consistently, it was reported that improvement of feed efficiency in pigs could be achieved by increasing lean growth rate, which resulted in lower feed intake ${ }^{[17]}$. Increasing dietary SAA showed no effect on carcass traits, which agreed well with previous studies ${ }^{[18]}$. 
In the present study, barrows showed lower shear force (a mechanical indicator of tenderness) than gilts, which agreed well with previous studies ${ }^{\text {[19] }}$. Meanwhile, it was also reported that tenderness scores were positively correlative with IMF content $(P=0.008)^{[19]}$. Consistent with these results, barrows also showed higher IMF in LM than gilts. Besides, it has been suggested that an increase in IMF leading to a decrease in the shear force could potentially be due to a decrease in the density of muscle which is positively correlated with protein content ${ }^{[20]}$. Consistent with this, barrows showed a lower crude protein content in LM compared with gilts, which also suggested the potential changes in the myofibrillar and cytoskeletal proteins correlating with shear force ${ }^{[21]}$. Mechanistically, IGF-1 has been shown to stimulate muscle protein synthesis and inhibit protein degradation via the ubiquitin-proteasome and autophagylysosome pathways ${ }^{[22]}$. Thus, the lower plasma IGF-1 concentration in barrows than in gilts provided physiological explanation for the reduced protein deposition and shear force.

The increase in dietary SAA as DL-Met or OH-Met tended to $(P=0.06)$ decrease the shear force of LM, which might also be explained by a tendency of a lower crude protein content in the LM of pigs fed increased SAA diets. As addressed above, the numerically lower plasma IGF-1 concentrations and, moreover, the down-regulation of IGF-1 expression in LM might in part account for the lower protein deposition in DL-Met- and OH-Met-fed pigs than in CON-fed pigs. Corresponding to the relatively lower crude protein deposition in LM, there was a reduction in crude protein of liver in pigs fed increased SAA diets, which might be associated with the limitation of circulating essential amino acids levels. In support of this, pigs fed DL-Met and OH-Met showed lower plasma histidine levels at 95-110 kg period compared with the CON (Additional file 3-5), but the mechanism for increased SAA-induced change of amino acids profile remains to be elucidated.

Remarkably, in comparison to the CON-fed pigs, the DL-Met- and OH-Met-fed pigs had increased glycolytic potential in LM. Generally, the higher level of glycolytic potential would increase drip loss and lead to pale, soft and exudative (PSE) pork. However, the decreased drip loss, in comparison to the control, excluded the occurrence of PSE pork in the DL-Met and OH-Met treatment. A moderately increased level of glycolytic potential was even considered to be beneficial for the development of tenderness ${ }^{[23]}$. Based on these results, we speculated that dietary DL-Met or OH-Met supplementation above growth requirements improved meat tenderness through changing protein and energy metabolism. Consistent with this assumption, the mRNA level of GLUT-4 and AMPKa2 in LM significantly increased in the DL-Met or OH-Met groups. AMPK can activate phosphorylase kinase, which then activates glycogen phosphorylase and promotes glycogenolysis ${ }^{[24]}$. GLUT-4, as the primary transporter of glucose uptake in the muscle, is up-regulated in the muscle upon activation of AMPK ${ }^{[25]}$. We also found the up-regulated expression of FATP-1 whereas the down-regulated expression of HSL in the DL-Met and OH-Met treatments compared with the CON treatment, further suggesting the activation of AMPK pathway ${ }^{[26]}$. In contrast, it has been reported that pigs (initial weight $\sim 105 \mathrm{~kg}$ ) fed diets supplemented with Met at 5 times the level of the control diet, for the last 14 days before slaughter, showed a tendency of reduced lipid content and glycolytic potential in the LM ${ }^{[27]}$. The long-term (20 weeks) supplementation and the lower increase (25\%) of Met applied in the current study might have led to different mechanisms of response. In support of this, following increased SAA consumption, plasma taurine levels were elevated from 11$70 \mathrm{~kg}$ period whereas it remained unchanged from $70-110 \mathrm{~kg}$ period, indicating the different responses of pigs varying in body weight to supplemental Met sources.

A decreased drip loss was observed in DL-Met- and OH-Met-fed pigs compared to the CON-fed pigs. As indicated by previous studies ${ }^{[27]}$, Met supplementation in pig diets improved the water-holding capacity (WHC) of LM, which was associated with the increased antioxidant capacity as manifested by the lower level of MDA in LM. However, the content of MDA in LM showed no difference among treatments in the present study. This might be associated with that Met

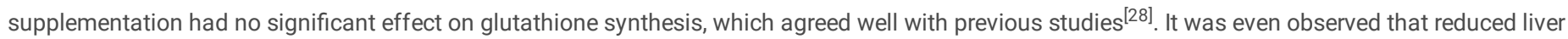
glutathione concentration following dietary supplementation with excessive cysteine ${ }^{[29]}$. These results suggested that the antioxidant capacity in response to SAA supplementation varied with the supplemental levels. Physically, drip loss originates from the spaces between muscle fiber bundles and the perimysial network, and the spaces between muscle fibers and the endomysial network ${ }^{[30]}$. Myofiber is constituted by four different fiber types including slow-oxidative or type I, fast oxido-glycolytic or type Ila, and fast glycolytic Ilx or Ilb. Glycolytic muscles had larger extra-myofibrillar fluid spaces and higher CSA than oxidative muscles $^{[31-32]}$. Moreover, drip loss was negatively related to fiber area percentages of type I and Ila, while positively related to type IIx and IIb percentage ${ }^{[33]}$. Interestingly, we found that the increase in dietary SAA decreased the CSA of myofibers and down-regulated mRNA levels of MyHC IIx. Mechanistically, AMPK can directly regulate the transcriptional activity of PPAR $\delta$ in skeletal muscles and thus increase the proportion of type I fibers (by $38 \%{ }^{[34]}$. Consistently, AMPKa2 in LM was up-regulated in the DL-Met and OH-Met groups in comparison to the CON group. Thus, it would appear that the decreased drip loss following increased SAA consumption was associated with the change of fiber types. In addition, we also found that drip loss was significantly affected by the interaction of diet and sex. Specifically, for the CON-fed pigs, drip loss of LM was higher in gilts than in barrows. As mentioned above, both IMF and marbling score were higher in barrows than in gilts. It was reported that marbling score was negatively correlated with drip loss $(r=-0.459 ; P=0.001)^{[35]}$. In the present study, the drip loss tended $(P=0.08)$ to be negatively correlated $(r=-0.52)$ with the IMF content. Thus, the higher IMF and marbling score in LM might account for the lower drip loss in the CON-fed barrows than in the CON-fed gilts.

Interestingly, the lightness of fresh muscle as indicated by $\mathrm{L}_{45 \mathrm{~min}}$ value was higher in the OH-Met-fed barrows than in the DL-Met- and CON-fed barrows. It has been reported that muscle lightness and plasma lactate and glucose reflect increased muscle glycogenolysis which, in turn, can explain the change of muscle lightness and plasma lactate and glucose levels ${ }^{[36]}$. Consistent with the higher lightness value, the $\mathrm{OH}$-Met treatment had higher degree of muscle glycogenolysis as suggested by the higher glycolytic potential. Moreover, the increase in the glycolytic potential in the $\mathrm{OH}-\mathrm{Met}$ treatment was mainly associated with the increased content of lactate, the end-product of glycolysis. In contrast, the increase in the glycolytic potential in the DL-Met treatment was mainly ascribed to the increase in free glucose and glucose-6-P content, the initial product of glycolysis. These results indicate that a higher degree of complete glycolysis process did occur in muscle of the $\mathrm{OH}$-Met treatment rather than in that of the DL-Met treatment. The highest lightness value, $\mathrm{L}_{45 \mathrm{~min}}$ observed in the barrows of the $\mathrm{OH}-M e t$ treatment was accompanied with the highest muscle lactate level, further suggesting the contribution of lactate to 
lightness value. Pigs fed OH-Met had higher serum acetate and propionate, both of which can stimulate GLP-1 secretion via activating the GPR43 receptors in intestinal L-cells ${ }^{[37]}$. Consistently, GPR43 expression was upregulated in LM and plasma GLP-1 concentration was increased in the OH-Met treatment. GLP-1 can increase glycogen synthase activity and stimulate both glucose oxidation and lactate formation ${ }^{[38]}$. Taken together, SCFA-induced GPR43/GLP1 signaling might account for the higher level of lactate in the $\mathrm{OH}-$ Met treatment.

\section{Conclusions}

In summary, the increase in dietary SAA levels by $25 \%$ over NRC recommendation showed a positive effect on pork quality. Specifically, the increased SAA consumption decreased drip loss and tended to decrease shear force. The trend of lower shear force of LM was associated with the decrease in crude protein and increase in glycolytic potential. Changes in drip loss was associated with the types of muscle fiber. The increased lightness value of fresh muscle from barrows fed OH-Met diets appeared to be associated with the increased lactate level, which can be further explained by the increased plasma SCFA concentrations, stimulated GPR43 expression and enhanced GLP-1 secretion.

\section{Abbreviations}

AA, amino acids; ADFI, average daily feed intake; ADG, average daily gain; AMPKa2, AMP-activated protein kinase a2; CON, control; CSA, cross sectional area; Cys, cysteine; FAS, fatty acid synthase; FATP-1, fatty acid transport protein - 1; G F, gain to feed; GLP-1, glucagon-like peptide 1; GLUT-4, glucose transporter-4; GPR43, G protein-coupled receptor 43; GPX, glutathione peroxidase; GSSG, glutathione disulfide; HSL, hormone-sensitive lipase; IGF-1, insulin growth factor 1; IGF-1R, IGF-1 receptors; IMF, Intramuscular fat; LM, Iongissimus lumborum muscle; MDA, malondialdehyde; Met, methionine; mTORC1, mechanistic target of rapamycin complex 1; MyHC, Myosin Heavy Chain; OH-Met, DL-2-hydroxy-4 (methylthio) butanoic acid; SAA, sulfur amino acids; SAM, S-adenosylmethionine; SCFA, short-chain fatty acids; SID-SAA, standard-ileal-digestible sulfur amino acids; T-GSH, total glutathione.

\section{Declarations}

\section{Authors' contributions}

The authors' contributions are as follows: $Z$ F, D A and $Y M$ conceived the present study and explained the data. PY and $S L$ conducted the study, analyzed the data and wrote the manuscript. D W, L C, B F, X Z, Y L, S X, J L and Y Z gave critical suggestions during experiment and writing the manuscript. J P, Y L, Y L, $P$ $\mathrm{W}, \mathrm{HZ}, \mathrm{XY}$ and $\mathrm{X} \mathrm{J}$ participated in the chemical analysis and collected the data. All the authors revised and approved the final manuscript.

\section{Ethics approval and consent to participate}

The protocol of this study was approved by the Animal Care and Use Committee of Animal Nutrition Institute, Sichuan Agricultural University, and was carried out in accordance with the National Research Council's Guide for the Care and Use of Laboratory Animals, Chinese Order No.676 of the State Council, date (1 March 2017).

\section{Consent for publication}

Not applicable

\section{Availability of data and materials}

\section{The datasets used and/or analysed during the current study are available from the corresponding author on reasonable request.}

\section{Competing interests}

The authors declare the following financial interests/personal relationships which may be considered as potential competing interests: Dolores I. BatononAlavo and Yves Mercier are employees of Adisseo (one of the financial supporters of the present study)

\section{Funding}

This work was supported by the Adisseo Life Science France SAS, Briand, Antony Cedex, France.

\section{Acknowledgements}

Not applicable 


\section{References}

1. NRC. Nutrient requirements of swine. National Academies Press: 2012.

2. Gu X, Orozco JM, Saxton RA, Condon KJ, Liu GY, Krawczyk PA, et al. SAMTOR is an S-adenosylmethionine sensor for the mTORC1 pathway. Science. 2017;358(6364):813-8.

3. Liu Y, Lv W, Yu B, Ju T, Yang F, Jiang M, et al. S-adenosylmethionine-induced adipogenesis is accompanied by suppression of Wnt/beta-catenin and Hedgehog signaling pathways. Mol Cell Biochem. 2013;382(1-2):59-73.

4. Castellano R, Perruchot MH, Tesseraud S, Metayer-Coustard S, Baeza E, Mercier Y, et al. Methionine and cysteine deficiencies altered proliferation rate and time-course differentiation of porcine preadipose cells. Amino Acids. 2017;49(2):355-66.

5. Zhang X, Li H, Liu G, Wan H, Mercier Y, Wu C, et al. Differences in plasma metabolomics between sows fed DL-methionine and its hydroxy analogue reveal a strong association of milk composition and neonatal growth with maternal methionine nutrition. Br J Nutr. 2015;113(4):585-95.

6. Canfora EE, Jocken JW, Blaak EE. Short-chain fatty acids in control of body weight and insulin sensitivity. Nat Rev Endocrinol. 2015;11(10):577-91.

7. AOAC. Official methods of analysis. Association of official analytical chemists 2000.

8. Ontiveros RR, Shermer WD, Berner RA. An HPLC method for the determination of 2-hydroxy-4-(methylthio)-butanoic acid (HMB) in supplemented animal feeds. Journal of Agricultural Food Chemistry. 1987;35(5):692-4.

9. Gu Y, Song Y, Yin H, Lin S, Zhang X, Che L, et al. Dietary supplementation with tributyrin prevented weaned pigs from growth retardation and lethal infection via modulation of inflammatory cytokines production, ileal expression, and intestinal acetate fermentation. J Anim Sci. 2017;95(1):226.

10. Li H, Wan H, Mercier Y, Zhang X, Wu C, Wu X, et al. Changes in plasma amino acid profiles, growth performance and intestinal antioxidant capacity of piglets following increased consumption of methionine as its hydroxy analogue. Br J Nutr. 2014;112(6):855-67.

11. NY/T 825. Technical regulation for testing of carcass traits in lean-type pig. In). Beijing: China Standard Press; 2004.

12. Walstra P, Merkus $G$ Procedure for assessment of the lean meat percentage as a consequence of the new EU reference dissection method in pig carcass classification: based on discussion in the EU Management Committee on Pig Meat and based on discussions with dissection experts during a meeting on May 18-19, 1994 at Zeist, NL; ID-DLO: 1996.

13. NPPC. (1999). National Pork Producers Council Marbling Standards. In). Des Moines, USA.

14. Zhang C, Luo J, Yu B, Zheng P, Huang Z, Mao X, et al. Dietary resveratrol supplementation improves meat quality of finishing pigs through changing muscle fiber characteristics and antioxidative status. Meat Sci. 2015;102:15-21.

15. Livak KJ, Schmittgen TD. Analysis of relative gene expression data using real-time quantitative PCR and the 2(-Delta Delta C(T)) Method. Methods. 2001;25(4):402-8.

16. Morales JI, Camara L, Berrocoso JD, Lopez JP, Mateos GG, Serrano MP. Influence of sex and castration on growth performance and carcass quality of crossbred pigs from 2 Large White sire lines. J Anim Sci. 2011;89(11):3481-9.

17. Saintilan R, Brossard L, Vautier B, Sellier P, Bidanel J, Van Milgen J, et al., Phenotypic and genetic relationships between growth and feed intake curves and feed efficiency and amino acid requirements in the growing pig. anima/2015, 9 (1), 18-27.

18. Htoo JK, Garbossa CA, Silveira H, Amaral LG, Barbosa NA, Cantarelli VS. 0976 Effects of methionine or arginine supplementation and environmental temperature on performance, carcass traits and meat quality of finishing pigs. J Anim Sci. 2016;94(suppl_5):469-.

19. Aaslyng MD, Jensen H, Karlsson AH. The gender background of texture attributes of pork loin. Meat Sci. 2018;136:79-84.

20. Karlsson AH, Klont RE, Fernandez X. Skeletal muscle fibres as factors for pork quality. Livestock Production Science 1999, 60 (2-3), 255 - 69.

21. Hopkins D, Geesink G. Protein degradation post mortem and tenderisation. Applied muscle biology and meat science $2009,149-73$.

22. Mammucari C, Milan G, Romanello V, Masiero E, Rudolf R, Del Piccolo P, et al. FoxO3 controls autophagy in skeletal muscle in vivo. Cell Metabol. 2007;6(6):458-71.

23. Lonergan EH, Zhang W, Lonergan SM. Biochemistry of postmortem muscle-Lessons on mechanisms of meat tenderization. Meat Sci. 2010;86(1):18495.

24. Liang J, Yang Q, Zhu M-J, Jin Y, Du M. AMP-activated protein kinase (AMPK) a2 subunit mediates glycolysis in postmortem skeletal muscle. Meat Sci. 2013;95(3):536-41.

25. Scheffler T, Gerrard D. Mechanisms controlling pork quality development: The biochemistry controlling postmortem energy metabolism. Meat Sci. 2007;77(1):7-16.

26. Li S, Wang H, Wang X, Wang Y, Feng J. Betaine affects muscle lipid metabolism via regulating the fatty acid uptake and oxidation in finishing pig. J Anim Sci Biotechnol. 2017;8:72.

27. Lebret B, Batonon-Alavo DI, Perruchot MH, Mercier Y, Gondret F. Improving pork quality traits by a short-term dietary hydroxy methionine supplementation at levels above growth requirements in finisher pigs. Meat Sci. 2018;145:230-7.

28. Green CO, Badaloo AV, Hsu JW, Taylor-Bryan C, Reid M, Forrester T, et al. Effects of randomized supplementation of methionine or alanine on cysteine and glutathione production during the early phase of treatment of children with edematous malnutrition. Am J Clin Nutr. 2014;99(5):1052-8.

29. Yin J, Ren W, Yang G, Duan J, Huang X, Fang R, et al. L-Cysteine metabolism and its nutritional implications. Mol Nutr Food Res. 2016;60(1):134-46.

30. Joo ST, Kim GD, Hwang YH, Ryu YC. Control of fresh meat quality through manipulation of muscle fiber characteristics. Meat Sci. 2013;95(4):828-36.

31. Polak JF, Jolesz FA, Adams DF. NMR of skeletal muscle. Differences in relaxation parameters related to extracellular/intracellular fluid spaces.

Investigative radiology. 1988;23(2):107-12. 
32. Schiaffino S, Reggiani C. Molecular diversity of myofibrillar proteins: gene regulation and functional significance. Physiological reviews. 1996;76(2):371423.

33. Ryu Y, Kim B. The relationship between muscle fiber characteristics, postmortem metabolic rate, and meat quality of pig longissimus dorsi muscle. Meat Sci. 2005;71(2):351-7.

34. Narkar VA, Downes M, Yu RT, Embler E, Wang YX, Banayo E, et al. AMPK and PPARdelta agonists are exercise mimetics. Cell. 2008;134(3):405-15.

35. Cannata S, Engle T, Moeller S, Zerby H, Radunz A, Green M, et al. Effect of visual marbling on sensory properties and quality traits of pork loin. Meat Sci. 2010;85(3):428-34.

36. Hemsworth PH, Barnett JL, Hofmeyr C, Coleman GJ, Dowling S, Boyce J. The effects of fear of humans and pre-slaughter handling on the meat quality of pigs. Aust J Agric Res. 2002;53(4):493-501.

37. Tolhurst G, Heffron H, Lam YS, Parker HE, Habib AM, Diakogiannaki E, et al. Short-chain fatty acids stimulate glucagon-like peptide-1 secretion via the Gprotein-coupled receptor FFAR2. Diabetes. 2012;61(2):364-71.

38. Villanueva-Peñacarrillo ML, Alcántara Al, Clemente F, Delgado E, Valverde I. Potent glycogenic effect of GLP-1(7-36)amide in rat skeletal muscle. Diabetologia. 1994;37(11):1163-6.

\section{Supplementary Files}

This is a list of supplementary files associated with this preprint. Click to download.

- Additionalfile3.docx

- Additionalfile3.docx

- Additionalfile2.docx

- Additionalfile2.docx

- Additionalfile1.docx

- Additionalfile1.docx 